\title{
Perda auditiva na hemoglobinopatia SC (HbSC): relato de caso
}

\author{
Hearing loss in SC hemoglobinopathy (HbSC): case report
}

\author{
Dourivaldo Silva Santos ${ }^{1}$, Luciene da Cruz Fernandes², Mara Renata Rissatto-Lago ${ }^{3}$, Caique dos Santos \\ Reis $^{4}$, Ana Caline Nóbrega da Costa ${ }^{5}$
}

\begin{abstract}
${ }^{1}$ Fonoaudiólogo. Mestre em Farmácia (PPgFAR/UFBA). Doutorando pelo Programa de Pós-Graduação em Processos Interativos de Órgãos e Sistemas do Instituto de Ciências da Saúde, UFBA; ${ }^{2}$ Doutora em Medicina e Saúde Pública pela Escola Bahiana de Medicina e Saúde Pública. Docente do Instituto de Ciências da Saúde, ICS/UFBA; ${ }^{3}$ Doutora

em Medicina e Saúde Pública pela Escola Bahiana de Medicina e Saúde Pública. Docente do Departamento de Ciências da Vida da Universidade do Estado da Bahia, UNEB; ${ }^{4}$ Discente do curso de Fonoaudiologia da Universidade do Estado da Bahia, UNEB; ${ }^{5}$ Doutora e Mestre em Medicina e Saúde, pela UFBA. Docente do Instituto de Ciências da Saúde, ICS/UFBA.
\end{abstract}

\begin{abstract}
Resumo
Introdução: Doença falciforme (DF) engloba um conjunto de hemoglobinopatias marcadas pela hemoglobina (Hb) anormal S (HbS). A $\mathrm{HbS}$ possui um formato de foice e aumento de rigidez, culminando em hemólise. Além disso, dificulta a passagem pela microcirculação sanguínea, causando vaso-oclusão e lesão isquêmica em diversos órgãos e tecidos. Na orelha interna, tem sido descrita como os responsável por danos auditivos. Objetivo: apresentar um relato de caso de paciente do sexo feminino com doença falciforme, acometida de perda auditiva sensorioneural (PASN) bilateral assimétrica. Relato do caso: paciente do sexo feminino, destra, 45 anos, compareceu para avaliação, queixando se de diminuição da audição e zumbido na orelha esquerda. Foi submetida à avaliação audiológica, constituída por audiometria tonal limiar, logoaudiometria, imitanciometria, emissões otoacústicas por produto de distorção (EOAPD) e potencial evocado auditivo do tronco encefálico (PEATE). Resultados: constatou-se: perda auditiva sensorioneural bilateral de grau leve na orelha direita e severo na orelha esquerda; presença dos reflexos estapedianos contralaterais na orelha direita e ausência na orelha esquerda; curvas timpanométricas tipo A; ausência de emissões otoacústicas bilateralmente; e os potenciais auditivos evocados do tronco encefálico dentro dos padrões de normalidade. Discussão: diversos mecanismos estão envolvidos na relação DF e PASN, como a falta de oxigenação e infarto do órgão de Corti, hemorragia labiríntica e labirintite ossificante, bem como uma associação entre o nível de viscosidade sanguínea, disfunção endotelial e hipertensão sistêmica. E ainda deve ser considerada a questão de dominância hemisférica na assimetria da perda. Conclusão: os conhecimentos sobre as características dos danos auditivos na HbSC ainda não são conclusivos e merecem mais investigações. A implementação de avaliações periódicas da função auditiva tem contribuído para prevenir a progressão dos danos e auxiliado no tratamento precoce.
\end{abstract}

Palavras-chave: Doença falciforme. Hemoglobinopatia SC. Vaso-oclusão. Avaliação auditiva. Perda auditiva sensorioneural.

\begin{abstract}
Introduction: Sickle cell disease (SCD) comprises a set of hemoglobinopathies marked by abnormal hemoglobin (Hb) S (HbS). HbS has a sickle shape and increased stiffness culminating in hemolysis, in addition to making it difficult to pass through the blood microcirculation, causing vessel-occlusion and ischemic damage in various organs and tissues. In the inner ear, they have been described as responsible for hearing damage. Objective: describing a case report of a female patient with sickle cell disease affected by asymmetric bilateral sensorineural hearing loss (SNHL). Case report: a 45 -year-old female patient, right-handed, came for evaluation, complaining of decreased hearing and tinnitus in her left ear. She underwent audiological evaluation consisting of pure tone audiometry, logoaudiometry, immittance testing, distortion product otoacoustic emissions (DPOAE) and brainstem auditory evoked potential $(B A E P)$. Results: bilateral mild sensorineural hearing loss in the right ear and severe in the left ear, presence of contralateral stapedial reflexes in the right ear and absence in the left ear, type A tympanometric curves, absence of bilateral otoacoustic emissions and the brainstem auditory evoked potentials within the normality patterns. Discussion: Several mechanisms are involved in the SCD and SNHL relationship, such as the lack of oxygen and infarction of the Organ of Corti, labyrinthine hemorrhage and ossifying labyrinthitis, as well as an association between the level of blood viscosity, endothelial dysfunction and systemic hypertension. And the question of hemispheric dominance in the loss asymmetry must still be considered. Conclusion: knowledge about the characteristics of hearing damage in $\mathrm{HbSC}$ is not yet conclusive and deserves further investigation. Implementation of periodic auditory function assessments has helped to prevent the progression of damage and has helped in early treatment.
\end{abstract}

Keywords: Sickle Cell Disease. Hemoglobinopathy SC. Vessel Occlusion. Audditive Evaluation. Sensorineural Hearing Loss.

Correspondente/Corresponding: *Ana Caline Nóbrega da Costa - Instituto de Ciência da Saúde, Universidade Federal da Bahia, Departamento de Fonoaudiologia - End: Av. Reitor Miguel Calmon, s/n, Canela Salvador/BA. - Tel: (71) 99975-9905 - E-mail: anacalinen@gmail.com

\section{INTRODUÇÃO}

Doença falciforme (DF) é um termo utilizado para englobar um grupo de hemoglobinopatias hereditárias, em que os glóbulos vermelhos assumem uma forma 
anormal, mais alongada, a hemoglobina S (HbS), em decorrência de mutações no gene que codifica a subunidade $\beta$ da hemoglobina (HB). As manifestações clínicas da DF ocorrem em indivíduos homozigóticos (HbSS) e em indivíduos heterozigotos nos quais existem associações de $\mathrm{HbS}$ com outras variantes de hemoglobinas, tais como $\mathrm{HbSC}, \mathrm{HbSD}$ e as interações com as talassemias (ALMEIDA; BERETTA, 2017; AZAR; WONG, 2017; GUALANDRO et al., 2015; POMPEO et al., 2020). No Brasil a prevalência do tipo HbSS varia de $1,2 \%$ a $10,9 \%$, e o tipo HbSC é relatado entre $0,15 \%$ e $7,4 \%$ dos casos de DF (KATO et al., 2018).

Em condições com pouca oxigenação, a hemoglobina $S$ entra em estado de polimerização, fazendo com que os glóbulos vermelhos percam sua forma arredondada e elástica e adquiram o aspecto de uma foice, tornando-se rígidos, com um risco elevado de ocorrer hemólise intravascular, o que causa a anemia hemolítica crônica (JOHN; JOHN, 2020). Esse formato dificulta a passagem do sangue pelos vasos de pequeno calibre, causando vaso-oclusão, lesão isquêmica e diminuição de oxigenação nos tecidos (SCHNOG; DUITS; MUSKIET, 2004). Além desses eventos crônicos, observam-se processos de dor aguda, disfunção endotelial vascular e aumento da atividade pró-inflamatória pela ativação de neutrófilos, monócitos e plaquetas, bem como a produção reduzida do óxido nítrico (SALINAS CISNEROS; THEIN, 2020).

A disfunção endotelial, os eventos de vaso-oclusão e a insuficiência circulatória no nível da orelha interna são os grandes responsáveis pela perda auditiva, devido à hipóxia ou ao infarto do órgão de Corti. Esses eventos alteram a atividade metabólica da estria vascular, afetando o equilíbrio iônico da endolinfa na orelha interna e causando morte das células ciliadas (LAGO et al., 2018; FARRELL et al., 2019). Além disso, o sistema nervoso é muito afetado com os baixos níveis de oxigênio, e isso implica um funcionamento anormal do nervo coclear e das vias auditivas centrais (JOVANOVIC-BATEMAN; HEDREVILLE, 2006). Também tem sido relatado que alguns fármacos empregados no tratamento dos pacientes com DF têm efeitos ototóxicos (KONTOGHIORGHES, 2007; STYLES; VICHINSKY, 1996).

Indivíduos com DF apresentam maior incidência de perda auditiva do que o resto da população, e PASN tem sido a mais relatada, com uma prevalência que varia de $3,8 \%$ a $21,4 \%$ entre crianças e jovens (MGBOR; EMODI, 2004) e de 46 a $66 \%$ entre adultos (DE CASTRO SILVA et al., 2010; ONAKOYA; NWAORGU; SHOKUNBI, 2002). Estudo de revisão sistemática com metanálise identificou uma ocorrência de 20,5\% (IC10,3-33\%) (RISSATTO-LAGO et al., 2019a). Além da perda auditiva, os reflexos acústicos estapedianos se apresentam alterados (PILTCHER et al., 2000; RISSATTO - LAGO et al., 2019b), e a ausência de EOAPD é relatada (BURCH-SIMS; MATLOCK, 2005; FARRELL et al., 2019). O aumento da amplitude das emissões otoacústicas pode indicar mudanças na micromecânica coclear e preceder a expressão detectável da PASN (RISSATTO-LAGO et al., 2019a). A avaliação do potencial evocado auditivo do tronco cerebral (PEATE) também apresenta alterações em pacientes com DF (DE CASTRO SILVA et al., 2010; JOVANOVIC-BATEMAN; HEDREVILLE, 2006; RISSATTO - LAGO et al., 2019b)

As perdas auditivas decorrentes da DF podem ocorrer tanto em crianças como em adultos, e diferentes padrões e graus de perda auditiva são relatados. A maioria apresenta severidade leve a moderada, acometendo uma ou ambas as orelhas e ambos os sexos. E também existe $o$ acometimento de perda auditiva de grau profundo registrado na literatura (BURCH-SIMS; MATLOCK, 2005; AL JABR, 2016; DA SILVA; NOVA; LUCENA, 2012; RISSATTO - LAGO et al., 2019a).

Aqui, relatamos o caso de uma paciente do sexo feminino, com diagnóstico de hemoglobinopatia SC (HbSC), acometida de perda auditiva sensorioneural bilateral assimétrica. A busca de evidências científicas que pudessem auxiliar na compreensão desse achado foi nosso objetivo.

\section{DESCRIÇÃO DO CASO}

A paciente foi atendida no ambulatório de diagnóstico audiológico para pacientes com doença falciforme da Universidade do Estado da Bahia (UNEB), proveniente do projeto de pesquisa aprovado pelo Comitê de Ética em Pesquisa parecer de número 3.194.367. Trata se de uma paciente de 45 anos, destra, sexo feminino, negra, com diagnóstico de hemoglobinopatia - SC (HbSC). Não fez o teste do pezinho, sendo diagnosticada na idade adulta, há mais de cinco anos. Apresentou o primeiro episódio vaso-oclusivo aos 42 anos, tendo três episódios, e não apresentou episódio nos últimos doze meses anteriores à avaliação auditiva. Não faz tratamento com hidroxiureia e nunca fez uso de transfusão exsanguínea. Os exames bioquímicos revelaram: hemoglobina $9,0 \mathrm{~g} / \mathrm{dL}$; hemoglobina fetal $2,5 \mathrm{~g} / \mathrm{dL}$; colesterol total $126,5 \mathrm{mg} / \mathrm{dL}$; HDL-C 41,2 $\mathrm{mg} / \mathrm{dL}$; LDL-C $61,0 \mathrm{mg} / \mathrm{dL}$; e glicose $96,0 \mathrm{mg} / \mathrm{dL}$.

Relata, como queixa auditiva, diminuição da audição de forma gradual, nos últimos cinco anos, na orelha esquerda, associada ao zumbido frequente de pitch grave, além da dificuldade para assistir TV. Não há casos de surdez na família. Apresenta hipertensão arterial sistêmica.

A avaliação audiológica foi realizada através da audiometria tonal limiar e vocal (limiar de reconhecimento de fala, LRF, e índice percentual de reconhecimento de fala, IPRF), avaliação da imitanciometria (timpanometria e reflexo acústico contralateral), pesquisa das emissões otoacústicas evocadas por produto de distorção (EOAPD); e a pesquisa do potencial evocado do tronco encefálico (PEATE).

A pesquisa do limiar auditivo foi realizada em audiômetro clínico, modelo Ad 229 (Interacoustic ${ }^{\circledR}$ ) e cabina acústica (calibração ANSI S3.6/96: ISSO 389/91. Foram pesquisadas as frequências de $0.25,0.5,1,2$, 4, 3, 6 e 8 $\mathrm{kHz}$. A timpanometria e a avaliação dos reflexos acústicos estapedianos (contralaterais nas frequências de 0.5, 1, 2 e $4 \mathrm{kHz}$ ) foram realizados no equipamento Otoflex Mad- 
$\operatorname{sen}^{\circledast}$. As respostas da EOAPD foram coletadas com o uso do equipamento OtoRead (Interacoustics ${ }^{\circledR}$ ), utilizando-se duas frequências primárias, $\mathrm{f} 1=65 \mathrm{~dB}$ SPL e f2 $=55 \mathrm{~dB}$ $\mathrm{SPL}$, com uma razão de frequência constante ( $\mathrm{f2} / \mathrm{f} 1$ ) de 1,2. As respostas foram pesquisadas nas frequências de 2, 3,4 e $5 \mathrm{kHz}$. Foi considerada como resposta quando a relação entre sinal e ruído foi $\geq 3 \mathrm{~dB}$.

O PEATE foi avaliado com equipamento Masbe ATC Plus (Contronic ${ }^{\circledR}$ ). 0 estímulo acústico utilizado foi o clique de $80 \mathrm{~dB}$ NA, monoaural, polaridade alternada, com 2.000 apresentações, com frequência equivalente a 17,2 cliques por segundo, filtro de banda de passa-alta em 100 $\mathrm{Hz}$, passa-baixo a $3000 \mathrm{~Hz}$ e janela de $10 \mathrm{~ms}$. As latências absolutas das ondas I, III e $\mathrm{V}$ e os intervalos interpicos de I-III, III-V, I-V foram analisados.

\section{RESULTADOS}

Os resultados obtidos na audiometria tonal limiar revelaram uma perda auditiva do tipo sensorioneural bilateral assimétrica, de grau leve na orelha direita e severo na orelha esquerda. Já os resultados do LRF e IPRF estão dentro do esperado e compatíveis, considerando-se os limiares tonais (Figura 1).

Figura 1 - Análise da audiometria tonal liminar e logoaudiometria

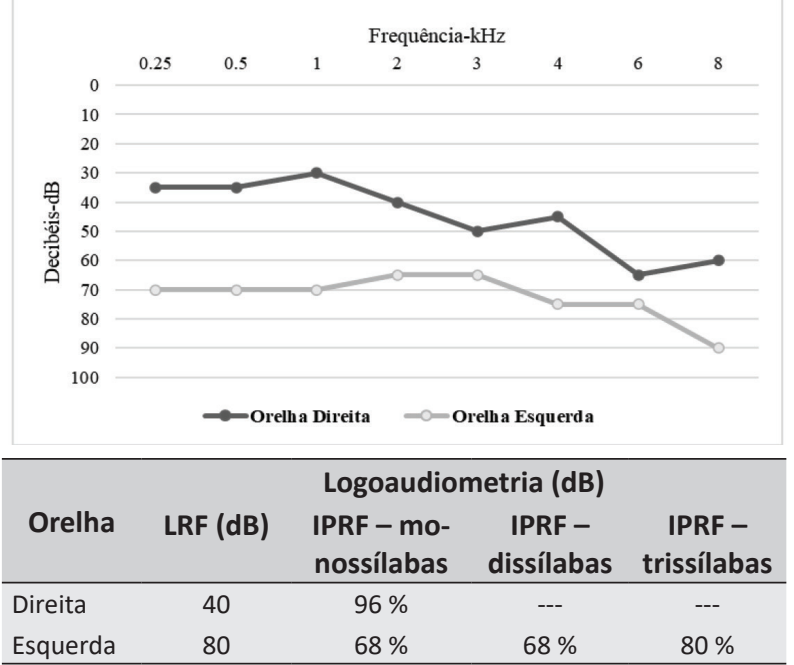

Legenda: LRF-limiar de reconhecimento de fala; IPRF-índice percentual de reconhecimento de fala.

Fonte: Dados da pesquisa

Os resultados da imitanciometria demonstram curvas timpanométricas tipo " $\mathrm{A}$ ", bilateralmente, e presença dos reflexos acústicos estapediano contralateral da orelha direita e ausência na orelha esquerda. As emissões otoacústicas por produto de distorção estavam ausentes em ambas orelhas (Tabela 1).
Tabela 1 - Analise da imitanciometria e EOAPD

\begin{tabular}{|c|c|c|c|}
\hline \multirow[t]{2}{*}{ Orelha } & Timpanometria & $\begin{array}{c}\text { RAE - Contrala- } \\
\text { teral }\end{array}$ & EOAPD \\
\hline & Curva & 0,5 a $4 \mathrm{kHz}$ & $2,3,4$ e $5 \mathrm{kHz}$ \\
\hline Direita & A & Presentes & Ausentes \\
\hline Esquerda & A & Ausentes & Ausentes \\
\hline
\end{tabular}

Legenda: RAE - reflexos acústicos estapedianos; EOAPD - emissões otoacústicas evocadas por produto de distorção.

Fonte: Dados da pesquisa

Os resultados do PEATE demonstraram latências absolutas e intervalos interpicos dentro dos padrões de normalidade bilateralmente, compatíveis com a integridade de condução eletrofisiológica da via auditiva (Tabela2).

Tabela 2 - Análise da pesquisa do potencial evocado auditivo do tronco encefálico

\begin{tabular}{lcccccc}
\hline \multirow{3}{*}{ Orelha } & \multicolumn{3}{c}{ Latências absolutas } & \multicolumn{3}{c}{ Latências interpicos } \\
& I & III & V & I-III & III-V & I-V \\
\hline Direita & 1,67 & 3,14 & 5,38 & 1,47 & 2,24 & 3,71 \\
Esquerda & 1,72 & 3,67 & 5,66 & 1,95 & 1,99 & 3,94 \\
\hline
\end{tabular}

Fonte: Dados da pesquisa

\section{DISCUSSÃO}

No caso relatado, a paciente de 45 anos, com diagnóstico de $\mathrm{HbSC}$, apresentou-se com queixas de hipoacusia em orelha esquerda, de forma gradual. Os resultados obtidos na audiometria tonal limiar revelaram uma perda auditiva do tipo sensorioneural bilateral assimétrica, de grau leve na orelha direita e severo na orelha esquerda, indicando que há um acometimento diferente entre as orelhas. As características da PASN ainda não estão bem estabelecidas na literatura com um padrão específico, sendo bem variadas. Farrel et al. (2019) relatam que, em 12 pacientes com PASN, a média dos limiares foram semelhantes nas duas orelhas, sugerindo que ambas as cócleas são igualmente afetadas (FARRELL et al., 2019). Ao se avaliarem 40 pacientes na faixa etária de 20 a 45 anos, 22,5\% foram diagnosticados com hipoacusia; ao verificar as médias dos tons puros, constatou-se não haver diferenças entre as orelhas direita e esquerda (AL JABR, 2016). Em 167 pacientes, a PANS foi registrada em 110 deles, com prevalência de $66 \%$, e 68 tinham perda auditiva bilateral. E observou-se que a dBNA média de ambas as orelhas piorava progressivamente, com o aumento da faixa etária (ONAKOYA; NWAORGU; SHOKUNBI, 2002).

$\mathrm{Na}$ fisiopatologia da DF que acomete o sistema auditivo, destacam-se os episódios vaso-oclusivos como um dos aspectos causadores da assimetria na perda auditiva sensorioneural, levando-se em consideração que os episódios acometem ambas as orelhas internas, porém com diferentes intensidades e graus de lesões. Mas vale salientar que, para afirmar, diante de uma avaliação, a 
patologia que desencadeia a perda auditiva assimétrica, é necessário utilizar, como recurso, exames de imagem complementares, como a ressonância magnética, o que não se torna acessível quando pensamos no alto custo de exames dessa complexidade (ALVES CARÇÃO et al., 2020). Ressaltamos que tem sido considerada a hipótese de que a perda de sangue durante os períodos menstruais pode levar a estresse hemodinâmico adicional e predispor a paciente a danos cocleares durante as crises vaso-oclusivas, piorando os limares adutivos (ADERIBIGBE; OLOGE; OYEJOLA, 2005).

Adicionalmente, têm sido propostos mecanismos específicos para justificar a PASN, como a hemorragia labiríntica e a labirintite ossificante, decorrentes de processos infecciosos inflamatórios ou destrutivos do labirinto membranoso (LIU et al., 2009). O aparecimento súbito de PANS e de sintomas otológicos, como zumbido, vertigem e tontura, parece estar mais associado aos quadros de hemorragia labiríntica, que afetam mais a região basal da cóclea e do vestíbulo (SAITO et al., 2011). A base da cóclea é responsável pelo processamento dos sons de altas frequências e, de fato, os estudos apontam que a perda auditiva decorrentes da DF afeta mais as altas frequências (DESAI; DEJOIE-BREWER; BALLAS, 2015; FARRELL et al., 2019; ONAKOYA; NWAORGU; SHOKUNBI, 2002).

Contudo, a irrigação coclear não occore somente no giro basal, mas se estende até a região apical, que também poderia estar sujeita a lesões na presença de crises vaso-oclusivas. No entanto, como a porção basal é conhecida por ser metabolicamente ativa, e comportar os principais receptores de energia acústica do ambiente externo, torna-se mais sensível às variações e (ou) à privação de oxigênio ou glicose. Portanto, mudanças em outras frequências sugerem que o dano é difuso na cóclea, em vez de limitado a seu giro basal (RISSATTO-LAGO et al., 11 2019a). Esses eventos poderiam justificar os diferentes graus de acometimento coclear e se manifestarem clinicamente em perdas auditivas assimetricas, como a observada no caso relatado.

Também tem sido considerada a hipótese de relação causal entre a hipertensão arterial sistêmica e a viscosidade sanguínea, que poderiam influenciar uma distribuição anormal de células falciformes pelo corpo. Tem sido observada maior viscosidade do sangue em pacientes com hipertensão sistêmica e DF (LEMONNE et al., 2014a). Com isso, a hiperviscosidade tem sido associada aos distúrbios otológicos em pacientes com $\mathrm{HbSC}$, e pode ser um fator predisponente para a surdez (DESAI; DEJOIE-BREWER; BALLAS, 2015). O aumento da viscosidade foi considerado o responsável pela isquemia no nível da artéria labiríntica, causando danos cocleares (JOHNSON, 2005; LEMONNE et al., 2014b).

A ausência das EOAPD, nesse caso, é um forte indicativo de danos nas células ciliadas externas, possivelmente pelos sofrimentos cocleares advindos de eventos vaso-oclusivos e hipóxia. Outros estudos observaram uma alta prevalência na redução das amplitudes das EOAPD em pacientes com DF (BURCH-SIMS; MATLOCK, 2005; FARRELL et al., 2019). Mesmo em crianças com limiares auditivos normais e função de orelha média íntegra, observou-se uma maior amplitudes das EOAPD naquelas com DF (WALKER; STUART; GREEN, 2004).

Os resultados obtidos com o PEATE estavam dentro dos padrões de normalidade, sugerindo que não havia comprometimento na condução da informação sonora nas vias auditivas centrais, reforçando o comprometimento no nível coclear. Mesmo as alterações observadas no exame PEATE, como as amplitudes reduzidas e (ou) as latências diminuídas, têm sido atribuídas a modulações no fluxo sanguíneo coclear (BURCH-SIMS; MATLOCK, 2005; DE CASTRO SILVA et al., 2010). E ainda, com base na literatura, podem-se indicar inúmeros indivíduos que participaram de estudos e que apresentaram perda auditiva assimétrica, o que caracteriza um fator comum entre as perdas auditivas. $\mathrm{E}$ um pequeno número desses pacientes com PASN tem patologias retrococleares (URBEN; BENNINGER; GIBBENS, 1999).

Além das questões auditivas relacionadas aos eventos da fisiopatologia da DF na orelha interna, outra hipótese a ser considerada para a assimetria observada no caso relatado seria a dominância da lateralidade hemisférica, já que se trata de um paciente destro. A assimetria hemisférica do sistema auditivo já é bem estabelecida, e muitos são os indícios de lateralização entre a cóclea e o córtex auditivo. A orelha esquerda possui um grau de desvantagem em relação à orelha direita e é mais suscetível a danos por ruído. Nela, o zumbido é mais predominante, e a perda auditiva mais frequente, ao passo que a sensibilidade auditiva é melhor na orelha direita (KHALFA et al., 1997).

Acredita-se no forte envolvimento do sistema olivococlear medial, pois, já que esse sistema desempenha um papel protetor, sua lateralização fisiológica pode ser relevante para a prevalência esquerda de zumbido e de fadiga auditiva. Desse modo, o sistema eferente olivococlear medial é mais eficaz na orelha direita, acreditando-se que isso decorre do fato de a orelha direita ser menos afetável na exposição ao ruído, por exemplo. Portanto, essas assimetrias do sistema auditivo eferente podem ser responsáveis pelas assimetrias de habilidade auditiva periféricas (KHALFA; VEUILLET; COLLET, 1998).

Ao ser empregado o teste de escuta dicótica, houve maior vantagem no reconhecimento de estímulos apresentados à orelha direita do que à esquerda, e essa destreza tem sido relacionada à vantagem da orelha direita (ILIADOU et al., 2010). Em adição, os estímulos tone burst monaurais, em adolescentes e adultos com audição normal, revelaram uma tendência de dominância hemisférica direita, caracterizando assimetrias corticais, independentemente da orelha estimulada. Esse padrão de respostas foi atribuído à organização anatômica das vias auditivas, nas quais a maioria dos neurônios aferentes se projetam da cóclea para o córtex auditivo contralateral (YAMAZAKI et al., 2017). 
Diante dos inúmeros mecanismos que poderiam estar afetando, direta ou indiretamente, a sensibilidade auditiva em pacientes com HbSC, o cenário ideal para um manejo clinico adequado deveria incluir avaliações com tomografia computadorizada e ressonância magnética, possibilitando um diagnóstico diferencial (SAITO et al., 2011), bem como monitorar os níveis de pressão arterial e oferecer tratamentos adequados (LEMONNE et al., 2014a). E, em alguns casos, seguir com a realização de flebotomia para evitar eventos agudos recorrentes e normalizar os níveis de hemoglobina, bem como realizar o monitoramento do nível de viscosidade no sangue (LEMONNE et al., 2014b).

Apesar de ser o relato apenas de um caso de PASN bilateral assimétrica, essa é uma oportunidade para investigar melhor os desfechos da HbSC no sistema auditivo. Os dados apresentados, juntamente com as evidências científicas para as perdas auditivas assimétricas, refletem a necessidade de mais investigações. Nesse sentido, torna-se necessária a condução de estudos controlados sobre incidência, prevalência, manifestações clínicas e fisiopatológicas das complicações da $\mathrm{HbSC}$ na sensibilidade auditiva, associados às comorbidades sistêmicas, como as da pressão arterial. Em adição, a implementação de modelos experimentais, incluindo a análise histopatológica e do estresse oxidativo, deverão auxiliar na compreensão desses fenômenos.

\section{CONCLUSÃO}

Com esse relato de caso, que evidencia uma perda auditiva sensorioneural bilateral assimétrica em uma paciente com $\mathrm{HbSC}$, demonstra-se que os conhecimentos sobre as características dos danos auditivos ainda não são conclusivos e merecem mais investigações, tendo em vista os inúmeros mecanismos envolvidos. Porém é indiscutível a necessidade de avaliações periódicas da função auditiva, para prevenir a progressão dos danos e auxiliar no tratamento precoce. Por fim, a avalição clínica - com inclusão de outros métodos, como a tomografia computadorizada, a ressonância magnética e o monitoramento dos níveis de viscosidade do sangue - auxiliaria na compreensão dos danos causados pela HbSC no sistema auditivo, possibilitando um controle mais adequado.

\section{REFERÊNCIAS}

ADERIBIGBE, A.; OLOGE, F. E.; OYEJOLA, B. A. Hearing thresholds in sickle cell anemia patients: emerging new trends? J. natl med. assoc., New York, v. 97, n. 8, p. 1135-1142, Ago. 2005.

AL JABR, I. Hearing loss among adults with sickle cell disease in an endemic region: a prospective case-control study. Ann. Saudi med., Riyadh, v. 36, n. 2, p. 135-138, 2016.

ALMEIDA, R. A. de; BERETTA, A. L. R. Z. Sickle Cell Disease and laboratory approach: a brief literature review. Rev. Bras. Anal. Clín., Rio de Janeiro, v. 49, n. 2, 2017.

ALVES CARÇÃO, A. et al. Assimetria auditiva prediz ressonância magnética alterada? Revista Portuguesa de Otorrinolaringologia e Cirurgia de Cabeça e Pescoço, [s.I], p. 127-130, 1 out. 2020.
AZAR, S.; WONG, T. E. Sickle Cell Disease. Med. clin. North America, Philadelphia, v. 101, n. 2, p. 375-393, mar. 2017.

BURCH-SIMS, G. P.; MATLOCK, V. R. Hearing loss and auditory function in sickle cell disease. J. commun. disord., v. 38, n. 4, p. 321-329, July 2005.

DA SILVA, L. P. A.; NOVA, C. V.; LUCENA, R. Sickle Cell anemia and hearing loss among children and youngsters: literature review. Braz. J. Otorhinolaryngol., São Paulo, v. 78, n. 1, p. 126-131, Jan. 2012.

DE CASTRO SILVA, I. M. et al. Auditory-evoked response analysis in Brazilian patients with sickle cell disease. Int. J. Audiol., Hamilton, v. 49, n. 4, p. 272-276, Jan. 2010.

DESAI, P.; DEJOIE-BREWER, M.; BALLAS, S. K. Deafness and sickle cell disease: three case reports and review of the literature. J. Clin. Med. Res., [s.I], v. 7, n. 3, p. 189-192, Mar. 2015.

FARRELL, A. N. et al. Sensorineural hearing loss in children with sickle cell disease. Int. j. pediatr. otorhinolaryngol., Amsterdam, v. 118, p. 110-114, Mar. 2019.

GUALANDRO, S. F. M. et al. Cohort study of adult patients with haemoglobin SC disease: clinical characteristics and predictors of mortality. Br. j. haematol., Oxford, v. 171, n. 4, p. 631-637, 2015.

ILIADOU, V. et al. Hemispheric laterality assessment with dichotic digits testing in dyslexia and auditory processing disorder. Int. j. audiol., Hamilton, v. 49, n. 3, p. 247-252, jan. 2010.

JOHN, N. A.; JOHN, J. E. Implications of COVID-19 infections in sickle cell disease. Pan Afr. med. j., [s.I], v. 36, 9 jun. 2020.

JOHNSON, C. S. Arterial blood pressure and hyperviscosity in sickle cell disease. Hematol. oncol. clin. north am., Philadelphia, v. 19, n. 5, p. 827-837, Oct. 2005.

JOVANOVIC-BATEMAN, L.; HEDREVILLE, R. Sensorineural hearing loss with BrainStem Auditory Evoked Responses changes in homozygote and heterozygote sickle cell patients in Guadeloupe (France). J. laryngol. otol., London, v. 120, n. 8, p. 627-630, Ago. 2006.

KATO, G. J. et al. Sickle cell disease. Nat. rev., Dis. primers, [s.I], v. 4, p. 18010, 2018.

KHALFA, S. et al. Evidence of peripheral hearing asymmetry in humans: clinical implications. Acta otolaryngol., Stockholm, v. 117, n. 2, p. 192196, Jan. 1997.

KHALFA, S.; VEUILLET, E.; COLLET, L. Influence of handedness on peripheral auditory asymmetry. Eur. J. Neurosci, Oxford, v. 10, n. 8, p. 2731-2737, Ago. 1998.

KONTOGHIORGHES, G. J. Deferasirox: uncertain future following renal failure fatalities, agranulocytosis and other toxicities. Exp. Opin. Drug Saf., [s.I], v. 6, n. 3, p. 235-239, May 2007.

LAGO, M. R. R. et al. Sensorineural hearing loss in children with sickle cell anemia and its association with endothelial dysfunction. Hematology, Amsterdam, v. 23, n. 10, p. 849-855, Dec. 2018.

LEMONNE, N. et al. Association between relative systemic hypertension and otologic disorders in patients with sickle cell-hemoglobin C disorder. Am. J. Hematol., New York, v. 89, n. 6, p. 667, 2014a.

LEMONNE, N. et al. Impaired blood rheology plays a role in the chronic disorders associated with sickle cell-hemoglobin $C$ disease. Haematologica, Pavia, v. 99, n. 5, p. 74-75, May 2014b.

LIU, B. P. et al. Labyrinthitis ossificans in a child with sickle cell disease: CT and MRI findings. Pediatr. radiol., Berlin, v. 39, n. 9, p. 999-1001, Sept. 2009. 
MGBOR, N.; EMODI, I. Sensorineural hearing loss in Nigerian children with sickle cell disease. Int. j. pediatr. otorhinolaryngol., Amsterdam, v. 68, n. 11, p. 1413-1416, Nov. 2004.

ONAKOYA, P. A.; NWAORGU, O. G. B.; SHOKUNBI, W. A. Sensorineural hearing loss in adults with sickle cell anaemia. Afr. j. med. med. sci., Oxford, v. 31, n. 1, p. 21-24, Mar. 2002.

PILTCHER, O. et al. Sensorineural hearing loss among sickle cell disease patients from southern Brazil. Am. J. Otolaryngol., Philadelphia, v. 21, n. 2, p. 75-79, abr. 2000.

POMPEO, C. M. et al. Fatores de risco para mortalidade em pacientes com doença falciforme: uma revisão integrativa. Esc. Anna Nery, Rio de Janeiro, v. 24, n. 2, 2020.

RISSATTO - LAGO, M. R. et al. Hidden hearing loss in children and adolescents with sickle cell anemia. Int. J. Pediatr. Otorhinolaryngol., Amsterdam, v. 116, p. 186-191, Jan. 2019b.

RISSATTO-LAGO, M. R. et al. Dysfunction of the auditory system in sickle cell anaemia: a systematic review with meta-analysis. Trop. med. int. health, Oxford, v. 24, n. 11, p. 1264-1276, 112019 a.

SAITO, N. et al. Clinical and radiologic findings of inner ear involvement in sickle cell disease. Am. J. Neuroradiology, Baltimore, v. 32, n. 11, p. 2160-2164, Dec. 2011.

SALINAS CISNEROS, G.; THEIN, S. L. Recent advances in the treatment of sickle cell disease. Front. Physiol., [s.I], v. 11, 20 may 2020.

SCHNOG, J. B.; DUITS, A. J.; MUSKIET, F. A. J. Sickle cell disease; a general overview. Neth j. med, Amsterdam, v. 62, n. 10, p. 11, 2004.

STYLES, L. A.; VICHINSKY, E. P. Ototoxicity in hemoglobinopathy patients chelated with desferrioxamine. J. pediatr. hematol. oncol., New York, v. 18, n. 1, p. 42-45, Feb. 1996.

URBEN, S. L.; BENNINGER, M. S.; GIBBENS, N. D. Asymmetric sensorineural hearing loss in a community-based population. Otolaryngol. head neck surg., Rochester, v. 120, n. 6, p. 809-814, June 1999.

WALKER, L. J.; STUART, A.; GREEN, W. B. Outer and middle ear status and distortion product otoacoustic emissions in children with sickle cell disease. Am. j. audiol., Rockville, v. 13, n. 2, p. 164-172, dec. 2004.

YAMAZAKI, H. et al. Cortical hemispheric asymmetries are present at young ages and further develop into adolescence. Hum. brain mapp., New York, v. 39, n. 2, p. 941-954, Nov. 2017.

Submetido em: $07 / 12 / 2020$

Aceito em: 14/12/2020 